\title{
Green Tea and Coronavirus Infection
}

\author{
Karimi Aliabadi Parastoo ${ }^{1}$, Sohrab Mehrnoush ${ }^{2}$, Zarrinpashneh Elham ${ }^{3}$ and Kashi Zahra ${ }^{4 *}$ \\ ${ }^{1}$ Department of Family Medicine, Mazandaran university of medical sciences, Iran \\ ${ }^{2}$ Department of Internal Medicine, diabetes research center, Mazandaran university of medical sciences, Iran \\ ${ }^{3}$ PharmD, Researcher, Iran
}

${ }^{4}$ Diabetes research center, Mazandaran university of medical sciences, Iran

*Corresponding author: Zahra Kashi, diabetes research center, Imam Khomeini hospital, Razi St., Sari, Iran

To Cite This Article: Karimi Aliabadi Parastoo, Sohrab Mehrnoush, Zarrinpashneh Elham, Kashi Zahra, Green Tea and Coronavirus Infection. 2020 - 10(5). AJBSR.MS.ID.001560. DOI: 10.34297/AJBSR.2020.10.001560.

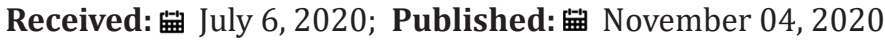

Keywords: Covid-19; Camellia Sinensis Complementary medicine

Abbreviations: SARS-CoV-2: Severe Acute Respiratory Syndrome Coronavirus-2; EC: Epicatechin; EGC: Epigallocatechin; ECG: Epicatechin-3gallate; EGCG: epigallocatechin-3-gallate; MERS-CoV: Middle Eastern Respiratory Syndrome Coronavirus; UPR: Unfolded Protein Response

\section{Introduction}

On the eve of 2020 the world was attacked by a new virus called Severe Acute Respiratory Syndrome Coronavirus-2 (SARS-CoV-2), which lead to a pneumonia called 'covid-19'. Within less than three months, several countries became infected with the virus and Globally, by the time of writing this manuscript, there have been $9,413,289$ confirmed cases of COVID-19, including 482,730 deaths, reported to WHO [1]. So far, no definitive treatment or confirmed vaccine has been found for this disease. COVID-19 and influenza viruses have similar disease presentations. They both cause multiorgan diseases especially involve respiratory system. The clinical manifestations of these viral infections may be mild or even asymptomatic to severe fatal illness [2]. Complementary medicine has long been considered for the treatment of diseases in Asian and Middle Eastern countries. Among them, drinking tea, has always been of interest to traditional healers.

\section{Effective ingredients in green tea}

Green tea (Camellia sinensis) has been shown to contain broad-spectrum antiviral effects against both DNA and RNA viruses [3]. Effective ingredients in green tea include the four main catechins: epicatechin (EC), epigallocatechin (EGC), epicatechin-3-gallate (ECG), and epigallocatechin-3-gallate (EGCG) [4].
EGCG, Quercetin and other catechins, are polyphenols known as flavonoids $[5,6]$. After ingestion of catechins, they undergo metabolic processing in the small intestine colon, and liver. This processing produces glucuronide and sulfate conjugates or methyl epicatechins [4]. Lim et al. demonstrated that green tea flavonoids have anti-inflammatory and anti-enzymatic activities by reducing NLRP3 inflammasome signaling, and consequently NFkB, TNF-a, IL-6, IL1B and IL-18 expression [7].

\section{Green tea and antiviral activity}

Thus far, there has been no in vitro or clinical studies specifically on the effects of green tea in prevention and treatment of SARSCoV2. However, previous studies demonstrated anti-viral effects of Green tea flavonoids in other members of coronavirus family and influenza viruses. Quercetin and EGCG were shown to inhibit the main protease of the SARS-CoV and Middle Eastern Respiratory Syndrome coronavirus (MERS-CoV) 3CLpro protease [8,9]. Moreover, Quercetin was demonstrated to modulate the cellular unfolded protein response (UPR) pathway and consequently inhibit coronavirus [10]. EGCG also exhibited antiviral activity by preventing the virus from entering the cell membrane in the early stages of infection $[6,11]$. In addition, it was shown that EGCG have antivi- 
ral effects on influenza virus subtypes by inhibiting its replication, RNA synthesis and agglutination in cell culture [12].

Clinical data also supported the anti-viral benefits of green tea. Noda et al. studied the effect of gargling green tea in 19,595 children. In this study, four subgroups of children gargled water, saline water, green tea, and functional water for twenty days. The rates of fever onset and sickness absence from the school were significantly lower in the green tea gargling group. They suggested that gargling with green tea had a great impact on the febrile disease caused by viral respiratory infections (e.g. rhinovirus, RS virus, coronavirus, adenovirus, and influenza virus) [13].

Yamada et al. also showed that gargling with the catechin extract solution, three times a day for three months, significantly lowered the influenza infection in the elderly, without any adverse events [14]. Another study by Mijong Park et al. demonstrated that consumption of 1-5 cups of green tea on an almost daily basis (i.e. $\geq 6 \mathrm{~d} / \mathrm{wk}$ ) reduced the incidence of influenza infection [15]. Matsumoto et al. designed a randomized, double-blind, placebo-controlled trial on 197 adult healthcare workers, consuming catechin/ theanine for five months. The incidence of clinically and laboratory influenza infection was significantly lower in the catechin/theanine group. They suggested that green tea supplements prevent infection with influenza virus [16].

\section{Conclusion}

Influenza epidemics occur in autumn and winter every year, and the end of Corona's pandemic is unknown [17]. In light of the previous studies demonstrating the beneficial effects of green tea in controlling the Influenza virus and SARS-CoV1, and genomic similarity between SARS-CoV1 and SARS-CoV2 [5], we highly recommend clinical studies to be conducted on the effects of green tea in prevention and treatment of Covid-19.

\section{References}

1. WHO Coronavirus Disease (COVID-19) Dashboard.

2. https://www.who.int/emergencies/diseases/novelcoronavirus-2019/question-and-ans wers-hub/q-adetail/q-a-similarities-and-differences-covid-19-and?gclid=EAIaIQobChMI8IujnuyZ6gIVCuAYCh03yAcsEAAYASAAEgKVavD_ BwE.
3. Xu J, Xu Z, Zheng WJM (2017) A review of the antiviral role of green tea catechins. Molecules 22(8): 1337.

4. Reygaert WC (2018) Green Tea Catechins: Their Use in Treating and Preventing Infectious Diseases. BioMed Research International :9105261.

5. Polansky H, Lori GJIJo AA (2020) Coronavirus (COVID-19), First Indication of Efficacy of Gene-Eden-VIR/Novirin in SARS-CoV-2 Infections. Int J Antimicrob Agents 55(6): 105971.

6. Alschuler L, Weil A, Horwitz R, Stamets P, Chiasson AM, et al. (2018) Integrative considerations during the COVID-19 pandemic. 20201550 8307(20): 30113-30120.

7. Lim H, Min DS, Park H, Kim HPJT (2018) Flavonoids interfere with NLRP3 inflammasome activation. Toxicol Appl Pharmaco 355: 93-102.

8. Nguyen TTH, Woo HJ, Kang HK, Kim YM, Kim DW, et al. (2012) Flavonoidmediated inhibition of SARS coronavirus 3C-like protease expressed in Pichia pastoris. Biochemistry 34(5): 831-838.

9. Park JY, Yuk HJ, Ryu HW, Lim SH, Kim KS, et al. (2017) Evaluation of polyphenols from Broussonetia papyrifera as coronavirus protease inhibitors. Journal of Enzyme Inhibition and Medicinal Chemistry 32(1): 504-512.

10. Nabirotchkin S, Peluffo AE, Bouaziz J, Cohen D (2020) Focusing on the unfolded protein response and autophagy related pathways to reposition common approved drugs against COVID-19.

11. Kaihatsu K, Yamabe M, Ebara YJM (2018) Antiviral mechanism of action of epigallocatechin-3-0-gallate and its fatty acid esters. 23(10): 2475.

12. Deryabin PG, Lvov DK, Botikov AG, Ivanov V, Kalinovsky T, et al. (2008) Effects of a nutrient mixture on infectious properties of the highly pathogenic strain of avian influenza virus A/H5N1. Biofactors 33(2): 85-97.

13. Noda T, Ojima T, Hayasaka S, Murata C, Hagihara A (2012) Gargling for oral hygiene and the development of fever in childhood: a population study in Japan. 22(1): 45-49.

14. Yamada H, Takuma N, Daimon T, Hara Y, Medicine C (2006) Gargling with tea catechin extracts for the prevention of influenza infection in elderly nursing home residents: a prospective clinical study. Controlled Clinical Trial 12(7): 669-672.

15. Park M, Yamada H, Matsushita K, Kaji S, Goto T, et al. (2011) Green Tea Consumption Is Inversely Associated with the Incidence of Influenza Infection among Schoolchildren in a Tea Plantation Area of Japan. J Nutr 141(10): 1862-1870.

16. Matsumoto K, Yamada H, Takuma N, Niino H, Sagesaka YMJBc (2011) Medicine a. Effects of green tea catechins and theanine on preventing influenza infection among healthcare workers: a randomized controlled trial. BMC Complement Altern Med. 11(1): 1-7.

17. The Flu Season. 